\title{
Sustained effect of continuous treatment with bevacizumab following bevacizumab in combination with chemotherapy in a human ovarian clear cell carcinoma xenograft model
}

\author{
NOBUYUKI ISHIKURA, KEIGO YOROZU, MITSUE KURASAWA, MIEKO YANAGISAWA, \\ MASAMICHI SUGIMOTO and KANAME YAMAMOTO
}

Product Research Department, Chugai Pharmaceutical Co., Ltd., Kamakura 247-8530, Japan

Received October 12, 2018; Accepted May 23, 2019

DOI: 10.3892/or.2019.7211

\begin{abstract}
Although bevacizumab maintenance following bevacizumab in combination with chemotherapy has demonstrated significant prolongation of progression-free survival in clinical studies in patients with ovarian cancer, the majority of the cancer cases in the study were of the serous histotype; therefore, data regarding clear cell carcinoma is limited. Furthermore, the efficacy of bevacizumab beyond progression has not yet been demonstrated in ovarian cancer. A xenograft model using the human ovarian clear cell carcinoma cell line RMG-I was used to investigate the antitumor effects and the mechanisms of bevacizumab in maintenance treatment and bevacizumab when administered beyond disease progression. In the RMG-I model, bevacizumab maintenance following bevacizumab in combination with paclitaxel exhibited increased tumor suppression, compared with its absence, and inhibited the increase of microvessel density (MVD) in tumors. Following disease progression during bevacizumab maintenance, continued bevacizumab treatment in combination with PEGylated liposomal doxorubicin as a secondary chemotherapeutic agent had increased efficacy, compared with PEGylated liposomal doxorubicin alone, and resulted in lower MVD accompanied with lower levels of insulin-like growth factor binding protein-3, which is reported to have angiogenic activity. Continuous suppression of angiogenesis by bevacizumab may contribute to the superior efficacy of bevacizumab maintenance and bevacizumab beyond progression in ovarian cancer.
\end{abstract}

Correspondence to: Dr Nobuyuki Ishikura, Product Research Department, Chugai Pharmaceutical Co., Ltd., 200 Kajiwara, Kamakura 247-8530, Japan

E-mail: ishikuranby@chugai-pharm.co.jp

Key words: bevacizumab, paclitaxel, PEGylated liposomal doxorubicin, maintenance therapy, bevacizumab beyond progression, angiogenesis, insulin-like growth factor binding protein-3, ovarian clear cell carcinoma

\section{Introduction}

Ovarian cancer accounted for $\sim 152,000$ mortalities annually globally in 2012 (1). Among the histological subtypes of ovarian cancer, ovarian clear cell carcinoma occurs more frequently in the Asian region, particularly in Japan (15-25\%), compared with North America and Europe (1-12\%), and is less sensitive to platinum-based chemotherapy with poorer prognosis, compared with serous adenocarcinoma (2-8). Therefore, more effective treatments are urgently required.

The GOG-0218 study demonstrated that progression-free survival (PFS) was significantly prolonged in patients with advanced epithelial ovarian cancer who received bevacizumab maintenance following induction therapy with carboplatin and paclitaxel plus bevacizumab, compared with PFS in patients who received induction therapy with carboplatin and paclitaxel plus bevacizumab without maintenance therapy, or in patients who received induction therapy with carboplatin and paclitaxel without maintenance therapy (9). These data resulted in the recommendation of the former regimen as the primary systemic therapy for patients with ovarian cancer with stage IV in guidelines, such as the NCCN guidelines (10). However, in that global study, the histotype in the majority of patients was serous carcinoma and the proportion of patients with clear cell carcinoma was as low as $2.9 \%$. Hence, there has been insufficient verification of the effectiveness against ovarian clear cell carcinoma of bevacizumab maintenance following induction therapy with carboplatin and paclitaxel plus bevacizumab.

Furthermore, although the effectiveness of continuous administration of bevacizumab in second-line treatment of patients with disease progression following combination therapy, including bevacizumab (bevacizumab beyond progression), has been demonstrated in metastatic colorectal cancer (11), the effectiveness of bevacizumab beyond progression has not been demonstrated in ovarian cancer.

Bevacizumab is a humanized monoclonal antibody against vascular endothelial cell growth factor (VEGF) that reduces abnormal blood vessels and inhibits VEGF-mediated angiogenesis in numerous cancer types, including colorectal cancer, renal cell carcinoma, non-small cell lung cancer, breast cancer, ovarian cancer and glioblastoma multiforme $(12,13)$. Paclitaxel is a taxane-based chemotherapeutic agent that stops the cell cycle 
by inhibiting tubulin depolymerization, thereby inhibiting cell division (14). It has been used in combination with carboplatin and bevacizumab in the primary treatment of metastatic ovarian cancer (10). Additionally, PEGylated liposomal doxorubicin, a formulation in which doxorubicin hydrochloride is encapsulated in polyethylene glycolated liposomes, has been used as monotherapy in recurrent ovarian cancer $(15,16)$.

In the present study, a human ovarian clear cell carcinoma xenograft model was used to investigate the antitumor effects and mechanisms of bevacizumab maintenance following induction treatment, including bevacizumab, and those of bevacizumab beyond progression.

\section{Materials and methods}

Antitumor agents. Bevacizumab was manufactured by Chugai Pharmaceutical Co., Ltd. (Tokyo, Japan) as a solution and diluted to $5 \mathrm{mg} / \mathrm{kg}$ body weight $(0.2 \mathrm{ml} / \mathrm{mouse})$ with saline. Human immunoglobulin $\mathrm{G}(\mathrm{HuIgG})$ was purchased from MP Biomedicals, LLC (Santa Ana, CA, USA), and was reconstituted with water and diluted to $5 \mathrm{mg} / \mathrm{kg}$ body weight (0.2 $\mathrm{ml} / \mathrm{mouse})$ with saline (Otsuka Pharmaceutical Co., Ltd., Tokyo, Japan). Paclitaxel was purchased from Wako Pure Chemical Industries, Ltd. (Osaka, Japan) and was reconstituted with 50\% ethanol-50\% Cremophor EL, and immediately prior to administration was diluted 10-fold with saline. PEGylated liposomal doxorubicin was purchased from Janssen Pharmaceutical, Inc. (Beerse, Belgium) as a solution and diluted to $20 \mathrm{mg} / \mathrm{kg}$ body weight $(10 \mathrm{ml} / \mathrm{kg}$ body weight) with saline.

Animals. A total of 908 female, 5-week-old BALB/c-nu/nu mice were purchased from Charles River Laboratories Japan, Inc. (Kanagawa, Japan) or CLEA Japan, Inc. (Tokyo, Japan). All animals were housed in a specific pathogen-free environment under controlled conditions (temperature, $20-26^{\circ} \mathrm{C}$; humidity, 35-75\%; 12/12 h light/dark cycle) and were allowed to acclimatize and recover from shipping-associated stress for $>6$ days prior to the study. Body weight of the mice at the time of randomization was 14.9-25.6 g. Chlorinated water and irradiated food were provided ad libitum. The health of the mice was monitored by daily observation. All animal experiments were reviewed and approved by the Institutional Animal Care and Use Committee at Chugai Pharmaceutical Co., Ltd. (approval no. 13-225, 15-201; Kamakura, Japan).

Cell lines and culture conditions. A total of two human ovarian cancer cell lines were used in the present study: SK-OV-3, which was established from the ascites fluid of a patient with ovarian serous adenocarcinoma and was demonstrated to be platinumsensitive (17); and RMG-I, which was established from the ascites fluid of a patient with ovarian clear cell carcinoma and was immunohistochemically confirmed to be clear cell carcinoma (18). SK-OV-3 cells were obtained from the American Type Culture Collection (Manassas, VA, USA) and were maintained by in vivo passage in BALB/c-nu/nu mice. RMG-I cells were obtained from National Institute of Biomedical Innovation (Osaka, Japan) and were maintained in Ham's F-12 Nutrient Mixture (Thermo Fisher Scientific, Inc., Waltham, MA, USA) supplemented with 10\% FBS (Bovogen Biologicals, Melbourne, Australia). All cells were cultured at $37^{\circ} \mathrm{C}$ in $5 \% \mathrm{CO}_{2}$.
In vivo tumor growth inhibition studies. Each BALB/c-nu/nu mouse was inoculated subcutaneously into the right flank with SK-OV-3 (8- $\mathrm{mm}^{3}$ tumor block) or RMG-I ( $5 \times 10^{6}$ cells). After 2-5 weeks of tumor inoculation, mice whose tumor had grown were randomly allocated to control, bevacizumab, paclitaxel, and paclitaxel plus bevacizumab induction treatment groups (week 1).

As the induction treatment in the SK-OV-3 and RMG-I xenograft models, HuIgG or bevacizumab $(5 \mathrm{mg} / \mathrm{kg}$, the maximum effective dose; intraperitoneally injected) and paclitaxel vehicle (5\% ethanol-5\% Cremophor EL-saline; intravenously injected) or paclitaxel $(40 \mathrm{mg} / \mathrm{kg}$, the optimum dose to evaluate the combination efficacy in the SK-OV-3 xenograft model, intravenously injected; $80 \mathrm{mg} / \mathrm{kg}$, the maximum tolerated dose, in the RMG-I xenograft model, intravenously injected) were administered on weeks 1,2 and 3.

On week 4, mice subjected to the induction treatment were subsequently subjected to control or bevacizumab maintenance treatment. When transitioning from a group of induction treatment to two groups of maintenance treatment, re-randomization was performed. As maintenance treatment, HuIgG or bevacizumab was administered weekly until 1 week prior to the date of the last tumor measurement (week 6, Fig. 1; week 9, Fig. 3B) or 1 day prior to the last tumor measurement (week 9, Fig. 3A).

For experiments of the second-line treatment, mice were treated with paclitaxel plus bevacizumab induction and then continuous bevacizumab maintenance, as aforementioned $(n=110)$. Tumor regrowth was defined as a consecutive increase in tumor volume exceeding $10 \%$ /week during bevacizumab maintenance. Mice bearing regrown tumors (with a volume of $240-720 \mathrm{~mm}^{3}$ ) were randomly allocated to each of the secondline control, bevacizumab, PEGylated liposomal doxorubicin, and PEGylated liposomal doxorubicin plus bevacizumab treatment groups ( $\mathrm{n}=7 /$ group). HuIgG or bevacizumab $(5 \mathrm{mg} / \mathrm{kg}$, the maximum effective dose; intraperitoneally injected) and PEGylated liposomal doxorubicin vehicle (saline; intravenously injected) or PEGylated liposomal doxorubicin $(20 \mathrm{mg} / \mathrm{kg}$, the maximum tolerated dose in the RMG-I xenograft model; intravenously injected) were administered, weekly and once every 3 weeks, respectively, until 1 week prior to the date of the last tumor measurement (week 15 in the second-line control or bevacizumab treatment groups; and week 22 in the secondline PEGylated liposomal doxorubicin or PEGylated liposomal doxorubicin plus bevacizumab treatment groups).

Tumor volume was measured once or twice a week. Tumor volume was estimated from the equation: Tumor volume $=a b^{2} / 2$, where $\mathrm{a}$ and $\mathrm{b}$ are tumor length and width, respectively. Individuals that succumbed due to accidental administration or individuals whose tumor self-destructed were excluded. The experimental protocol mandated that tumor volume measurement in both the second-line control group and the second-line bevacizumab group was to end whenever tumor self-destruction or blister breakage was observed in three or more mice in the second-line control or second-line bevacizumab groups. When tumor measurement was terminated by blister breakage, tumor samples were collected within 4 days from a site that was less affected by blister breakage.

Immunohistochemistry and quantification of microvessel density (MVD) in tumor tissues. Immunohistochemical analyses of 
CD31 was conducted using a Rat Horseradish Peroxidase (HRP)Polymer 1-Step (Mouse adsorbed) system (cat. no. BRR4016; Biocare Medical, LLC, Pacheco, CA, USA) according to the manufacturer's protocols. Subsequently, 5- $\mu \mathrm{m}$ thick sections from optimal cutting temperature compound-embedded freshly frozen tissues were fixed in $4 \%$ paraformaldehyde at $4{ }^{\circ} \mathrm{C}$ for $10 \mathrm{~min}$. The endogenous peroxidase activity and endogenous nonspecific background were blocked with $0.3 \%$ hydrogen peroxide at room temperature for $30 \mathrm{~min}$ and Rodent Block M (undiluted; cat. no. RBM961, Biocare Medical, LLC) at room temperature for $30 \mathrm{~min}$. The tissue sections were incubated at $4^{\circ} \mathrm{C}$ overnight with rat anti-mouse CD31 monoclonal antibody (clone MEC 13.3; 1:500; cat. no. 553370, BD Biosciences; Becton-Dickinson and Company, Franklin Lakes, NJ, USA) as the primary antibody. Subsequently, the sections were incubated at room temperature with the Rat HRP-Polymer 1-Step (Mouse adsorbed; undiluted) for $30 \mathrm{~min}$. Staining was conducted at room temperature using 3,3'-diaminobenzidine solution (DAB+, Liquid, 2-component system; cat. no. K3468; Agilent Technologies, Inc., Santa Clara, CA, USA) for $5 \mathrm{~min}$. All sections were counterstained at room temperature with hematoxylin for $2 \mathrm{sec}$. Tumor samples from freshly frozen tissues were collected on weeks 6, 9, 16 and 22. MVD (\%) was calculated from the ratio of the CD31-positive staining area to the total observation area in the viable region. A total of 3-6 fields/section were analyzed at a magnification of x100 with a light microscope (ECLIPSE Ni; Nikon Corporation, Tokyo, Japan), excluding necrotic areas. Positive-staining areas were calculated using imaging analysis software (WinROOF Version 6.3.1; Mitani Corporation, Fukui, Japan).

Human angiogenesis array and human insulin like growth factor binding protein-3 (IGFBP-3) ELISA assay. Tumor tissues collected from xenografts and stored at $-80^{\circ} \mathrm{C}$ were homogenized with Cell Lysis buffer (Cell Signaling Technology, Inc., Danvers, MA, USA) with Complete Protease Inhibitor Cocktail Tablets and Complete Phosphatase Inhibitor Cocktail Tablets (both from Roche Diagnostics, Basel, Switzerland). The homogenate was centrifuged at $9,100 \mathrm{xg}$ at $4^{\circ} \mathrm{C}$ for $20 \mathrm{~min}$. The resultant supernatant was used for the assays as cell lysates. Protein concentration of the cell lysates was quantified using a Direct Detect spectrometer (Merck KGaA, Darmstadt, Germany). Human Angiogenesis Array and Human IGFBP-3 ELISA (both from R\&D Systems, Inc., Minneapolis, MN, USA) were performed using the cell lysates. ImageQuant 400 (GE Healthcare Life Sciences, Little Chalfont, UK) was used for detection of the Human Angiogenesis Array.

Statistical analysis. Data were presented as mean + standard deviation, and analyzed with the Wilcoxon test. For comparison between the two groups, $\mathrm{P}<0.05$ was considered to indicate a statistically significant difference. For multiple comparison, the P-values were corrected by the Holm-Bonferroni method (19). All statistical analyses were conducted using JMP software (SAS Institute, Inc., Cary, NC, USA).

\section{Results}

Antitumor activity of bevacizumab maintenance treatment following paclitaxel plus bevacizumab induction treatment. Firstly, the efficacy of bevacizumab treatment in the induction
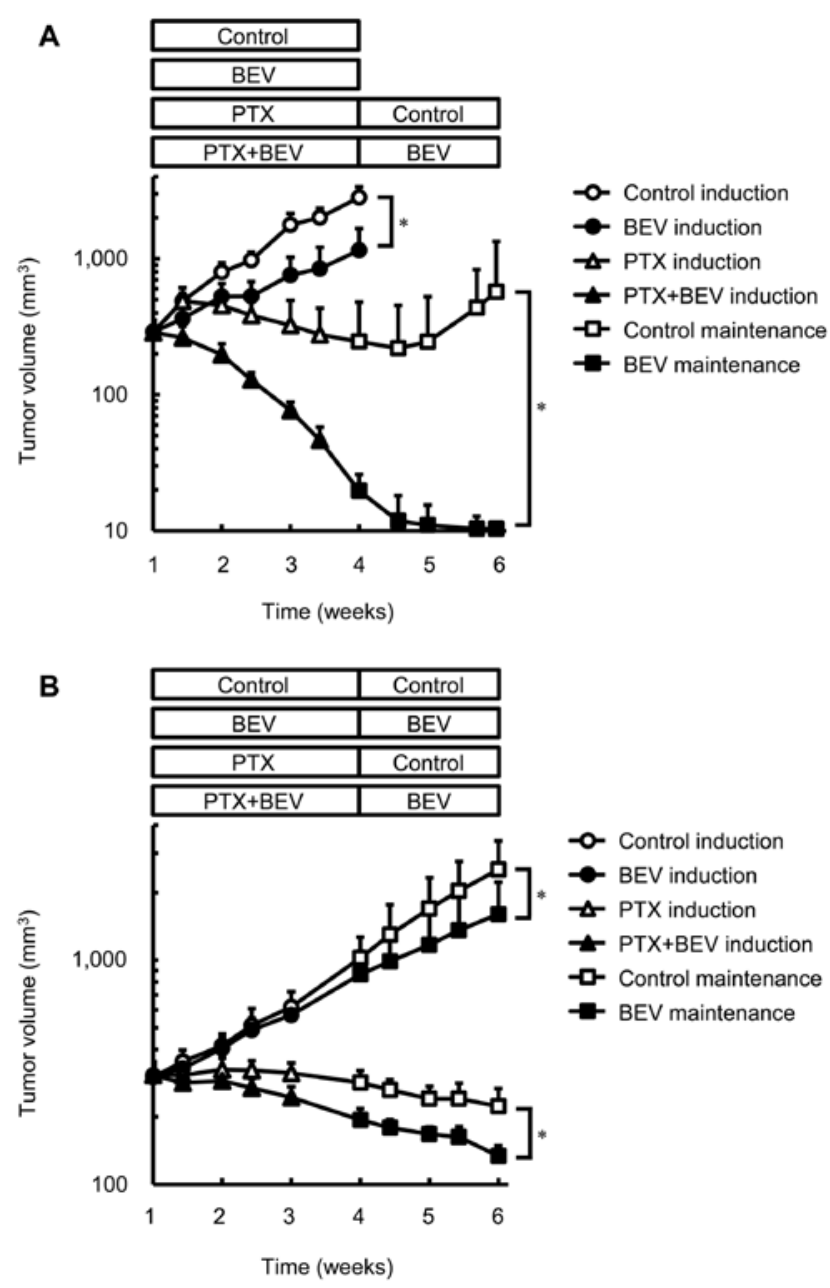

Figure 1. Antitumor activity of BEV maintenance following PTX plus BEV induction treatment in SK-OV-3 and RMG-I xenograft models. (A) Mice bearing SK-OV-3 tumors were randomly divided into four groups ( $\mathrm{n}=6$ /group) and were treated with control (vehicle for PTX or HuIgG for BEV), BEV, PTX or $\mathrm{PTX}+\mathrm{BEV}$ on weeks 1,2 , and 3 as induction treatment, and subsequently treated weekly with control or BEV as maintenance treatment. The control induction group and BEV induction group were examined until week 4 , as scheduled. The dosage of PTX and BEV was 40 and $5 \mathrm{mg} / \mathrm{kg}$, respectively. (B) Mice bearing RMG-I tumors were randomly divided into four groups and treated with control (vehicle for PTX or HuIgG for BEV), BEV, PTX or PTX+BEV on weeks 1, 2, and 3 as induction treatment, and subsequently treated weekly with control or $\mathrm{BEV}$ as maintenance treatment $(\mathrm{n}=6-7)$. The dosage of PTX and BEV was 80 and $5 \mathrm{mg} / \mathrm{kg}$, respectively. A total of one mouse with intraperitoneal tumor in the BEV maintenance following BEV induction treatment group was excluded from data analysis. Data points represent the mean + standard deviation of tumor volume $\left(\mathrm{mm}^{3}\right)$. * $\mathrm{P}<0.05$, Wilcoxon test $(\mathrm{B})$ with or $(\mathrm{A})$ without HolmBonferroni correction. BEV, bevacizumab; PTX, paclitaxel.

and maintenance phases in the human ovarian serous carcinoma cell line SK-OV-3 xenograft model and in the human ovarian clear cell carcinoma cell line RMG-I xenograft model was evaluated. In the SK-OV-3 xenograft model, tumor volume in the bevacizumab maintenance following paclitaxel plus bevacizumab induction treatment group on week 6 was significantly reduced, compared with the control maintenance following paclitaxel induction treatment group (Fig. 1A). A similar effect was observed in the RMG-I xenograft model, with the tumor volume in the bevacizumab maintenance following paclitaxel plus bevacizumab induction group on week 6 being significantly reduced, compared with the control maintenance following paclitaxel induction group (Fig. 1B), indicating that the addition 
A

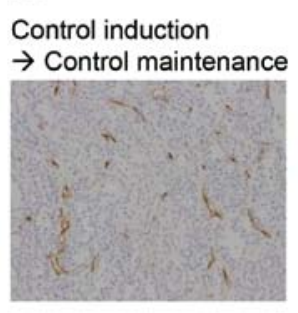

B

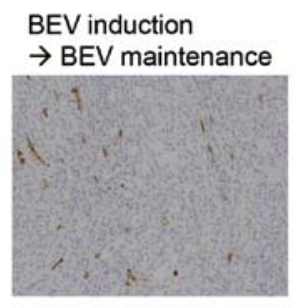

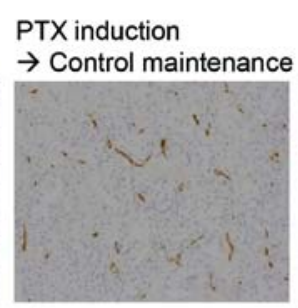

PTX+BEV induction $\rightarrow$ BEV maintenance

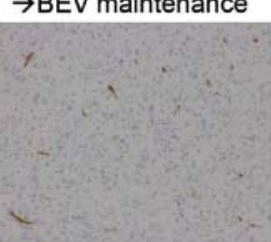

$-100 \mu \mathrm{m}$

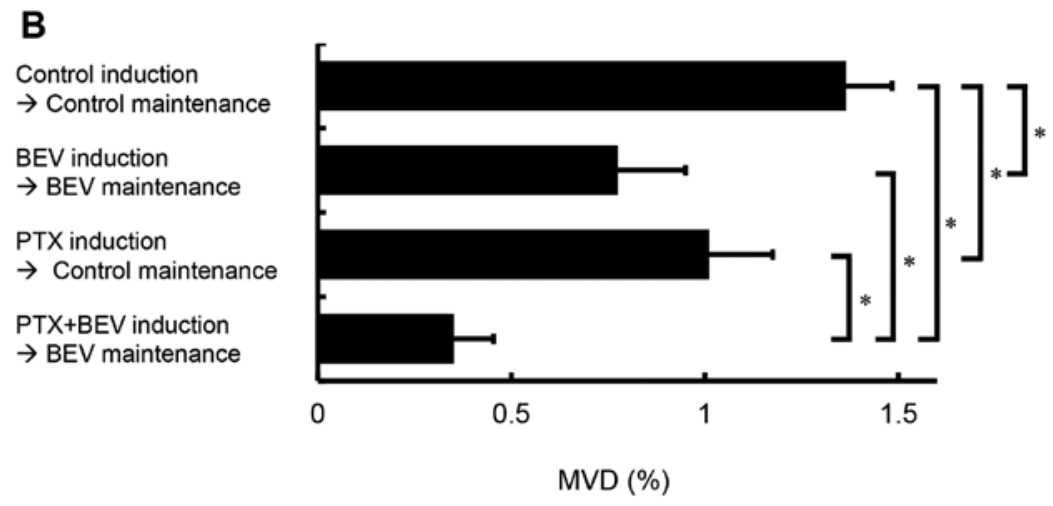

Figure 2. Anti-angiogenic activity of BEV maintenance following PTX plus BEV induction treatment in the RMG-I xenograft model. (A) Tumor microvessels stained immunohistochemically with antibody against CD31 on week 6. (B) MVD in tumor tissue was determined by calculating the ratio of CD31-positive area to the total observed area. The dosage of PTX and BEV was 80 and $5 \mathrm{mg} / \mathrm{kg}$, respectively. Data represent the mean + standard deviation ( $\mathrm{n}=6-7)$. ${ }^{*} \mathrm{P}<0.05$, Wilcoxon test with Holm-Bonferroni correction. BEV, bevacizumab; PTX, paclitaxel; MVD, microvessel density.

of bevacizumab during and following paclitaxel induction was notable in ovarian serous and clear cell carcinoma models.

Anti-angiogenic activity of bevacizumab maintenance treatment following paclitaxel plus bevacizumab induction treatment in the RMG-I xenograft model. To estimate the angiogenesis inhibitory activity, the MVD in RMG-I tumor tissues was evaluated from specimens obtained on week 6 . The MVD on week 6 was significantly lower in the bevacizumab maintenance treatment following bevacizumab induction treatment group and in the control maintenance treatment following paclitaxel induction treatment group, compared with the control maintenance following control induction treatment group (Fig. 2). Additionally, the MVD on week 6 was significantly lower in the bevacizumab maintenance following paclitaxel plus bevacizumab induction group, compared with the three aforementioned treatment groups.

Comparison of antitumor activity of bevacizumab maintenance treatment vs.control maintenance treatment following paclitaxel plus bevacizumab induction treatment in the RMG-I xengoraft model. Subsequently, the effect of bevacizumab treatment in the maintenance phase in the RMG-I xenograft with a longer treatment duration than in Fig. 1B was evaluated. The tumor volume in the bevacizumab maintenance treatment group following paclitaxel plus bevacizumab induction treatment was significantly reduced on week 9 , compared with the tumor volume in the control maintenance groups following the same induction treatment of paclitaxel induction treatment. In the bevacizumab maintenance treatment following paclitaxel plus bevacizumab induction treatment group, the tumor volume was significantly reduced on week 4 (start date of maintenance treatment), compared with week 1 (start date of induction treatment), and the tumor volume was significantly reduced on week 9, compared with week 4, indicating that the tumors in this group continued to significantly regress not only during induction treatment, but also during bevacizumab maintenance treatment (Fig. 3A).

Comparison of antitumor activity of bevacizumab maintenance treatment following paclitaxel plus bevacizumab induction treatment vs. bevacizumab maintenance treatment following paclitaxel induction treatment in the RMG-I xenograft model. Subsequently, the effect of bevacizumab treatment in the induction phase in the RMG-I xenograft with bevacizumab maintenance treatment was evaluated. Tumor volume in the bevacizumab maintenance treatment following paclitaxel plus bevacizumab induction treatment group was significantly reduced, compared with the bevacizumab maintenance following paclitaxel induction treatment group on week 9, indicating the potency of bevacizumab induction treatment (Fig. 3B).

Comparison of anti-angiogenic activity of bevacizumab maintenance treatment vs. control maintenance treatment following paclitaxel plus bevacizumab induction treatment in the RMG-I xenograft model. The MVD in RMG-I tumor tissues on week 9 in the bevacizumab maintenance treatment following paclitaxel plus bevacizumab induction treatment group was significantly lower, compared with the control maintenance following paclitaxel plus bevacizumab induction treatment group (Fig. 3C and D).

Antitumor activity of second-line PEGylated liposomal doxorubicin plus bevacizumab treatment following paclitaxel plus bevacizumab induction treatment and bevacizumab 
A
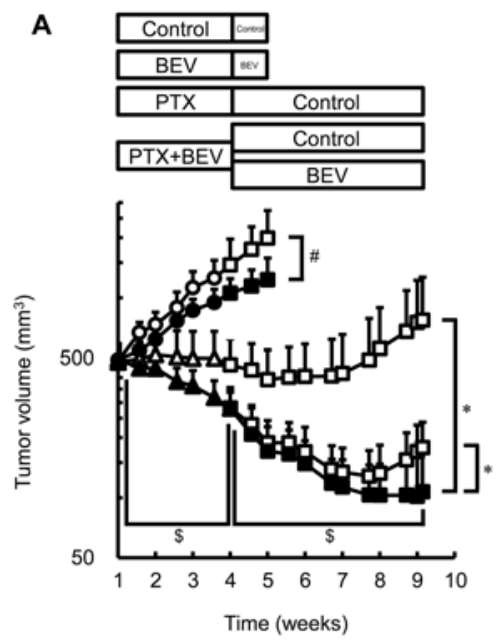

B

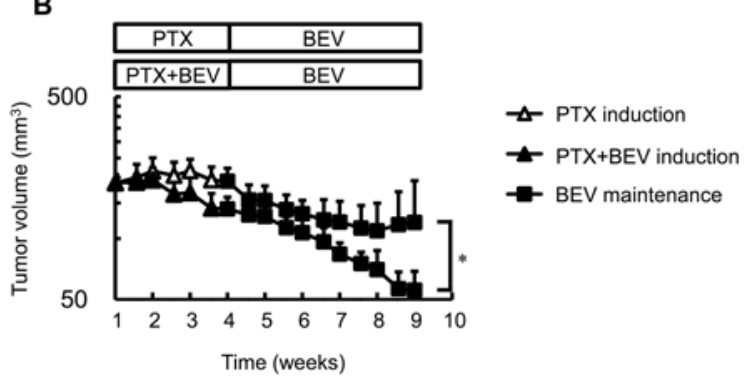

C PTX+BEV induction PTX+BEV induction $\rightarrow$ Control maintenance $\rightarrow \mathrm{BEV}$ maintenance

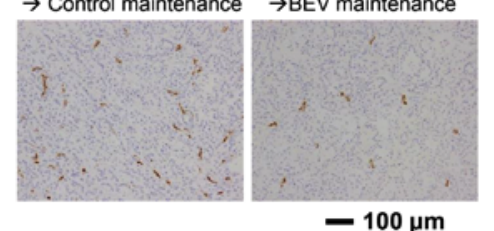

D

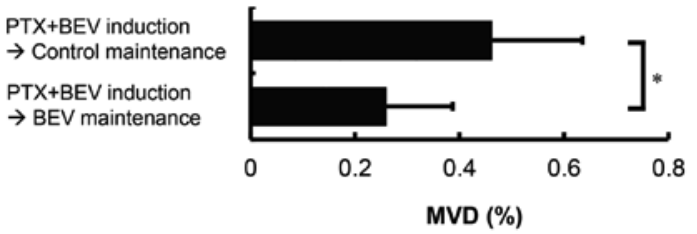

Figure 3. Antitumor activity of BEV maintenance treatment vs control maintenance treatment following PTX plus bevacizumab induction treatment in the RMG-I xenograft model. (A) Mice bearing RMG-I tumors were randomly divided into four groups: Control induction group $(n=7)$; BEV induction group $(\mathrm{n}=7)$; PTX induction group $(\mathrm{n}=7)$; and PTX+BEV induction group $(\mathrm{n}=16)$, and were treated with control (vehicle for PTX or HuIgG for BEV), BEV, PTX or PTX+BEV, respectively, on weeks 1, 2, and 3. On week 4, mice receiving $\mathrm{PTX}+\mathrm{BEV}$ induction treatments were re-randomized into a control maintenance and a BEV maintenance group ( $n=8$ per group). Mice were subsequently treated weekly with control (HuIgG) or BEV as maintenance treatment. Data points represent the mean + standard deviation of tumor volume $\left(\mathrm{mm}^{3}\right)$. ${ }^{*} \mathrm{P}<0.05$ and ${ }^{\$} \mathrm{P}<0.05$, Wilcoxon test with Holm-Bonferroni correction; ${ }^{\text {\# }} \mathrm{P}<0.05$, Wilcoxon test. (B) Mice bearing RMG-I tumors were randomly divided into two groups: PTX induction group; and PTX+BEV induction group, and were treated with PTX or PTX+BEV, respectively, on weeks 1, 2, and 3. From week 4, mice receiving induction treatments were subsequently treated weekly with $\mathrm{BEV}$ as maintenance treatment $(n=6-7)$. A total of one mouse was accidentally administered in the BEV maintenance following PTX+BEV induction treatment group was excluded from data analysis on week 4 . Data points represent the mean + standard deviation of tumor volume $\left(\mathrm{mm}^{3}\right)$. "P<0.05, Wilcoxon test. (C) Tumor microvessels stained immunohistochemically with antibody against CD31 on week 9. (D) The MVD in tumor tissue was determined by calculating the ratio of CD31-positive area to the total observed area. The dosage of PTX and BEV was 80 and $5 \mathrm{mg} / \mathrm{kg}$, respectively. Data represent the mean + standard deviation $(\mathrm{n}=6-7) .{ }^{*} \mathrm{P}<0.05$, Wilcoxon test. BEV, bevacizumab; PTX, paclitaxel; MVD, microvessel density.
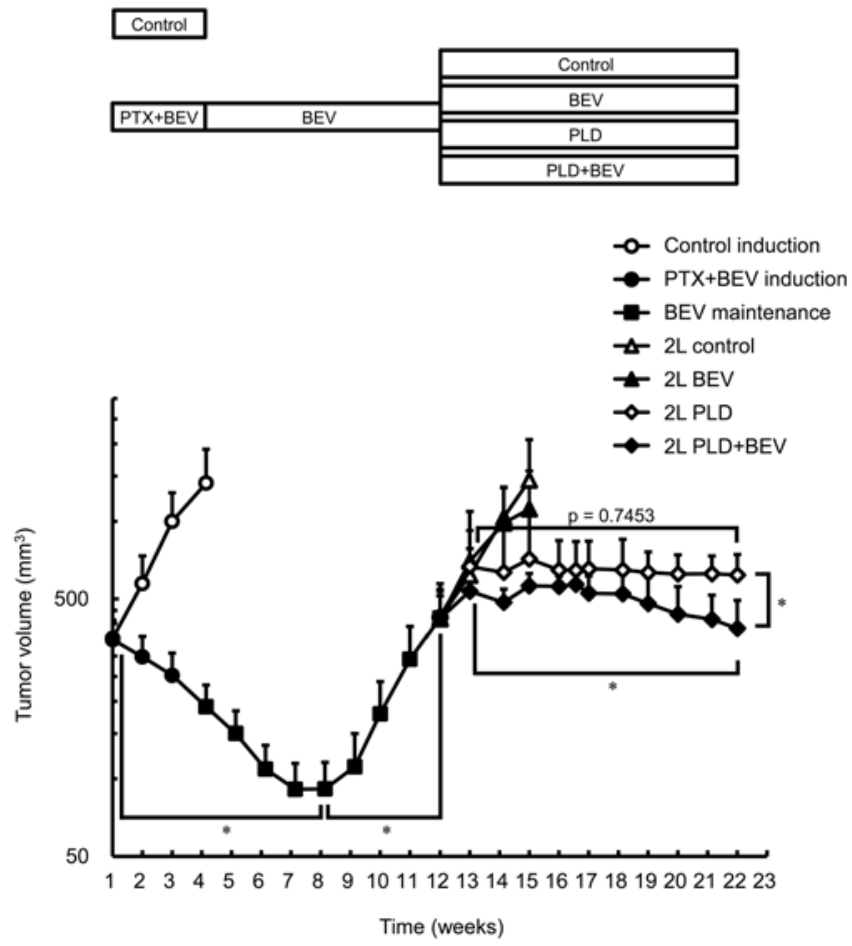

Figure 4. Antitumor activity of 2L PLD plus BEV treatment following PTX plus $\mathrm{BEV}$ induction treatment and BEV maintenance treatment in the RMG-I xenograft model. Mice bearing RMG-I tumors were randomly divided into a control induction group $(\mathrm{n}=6)$ and a PTX+BEV induction and $\mathrm{BEV}$ maintenance group $(\mathrm{n}=110)$ and treated with control (vehicle for PTX plus HuIgG) or PTX+BEV on weeks 1,2 , and 3 as first-line induction treatment, and subsequently treated weekly with BEV as maintenance treatment. The control induction group was examined until week 4 , as scheduled. On week 12 , mice treated with BEV maintenance and whose tumors had regrown were selected and re-randomized into four groups (n=7/group): 2L control group; BEV group; PLD group; and PLD+BEV group. At re-randomization, 82 mice were excluded, including individuals whose tumor did not regrow or those that were excluded to reduce the variability in tumor volume, body weight and growth rate. Plots of the PTX+BEV induction and BEV maintenance group were based on 28 individuals selected on week 12. Mice were subsequently treated with PLD once every 3 weeks and/ or BEV weekly as the $2 \mathrm{~L}$ treatment. The dosage of PLD, BEV and PTX was 20 , 5 and $80 \mathrm{mg} / \mathrm{kg}$, respectively. The following individuals receiving $2 \mathrm{~L}$ treatment were excluded from data analysis: 1 mouse with tumor self-destruction in the 2L BEV group on week 13; 1 mouse with tumor blister breakage in the 2L BEV group on week 14; 1 mouse that was accidentally administered in the 2L PLD group on week 19; 1 mouse with tumor self-destruction in the 2L PLD group on week 19; and 1 mouse that was accidentally administered in the 2L PLD+BEV group on week 13. Data points represent the mean + standard deviation of tumor volume $\left(\mathrm{mm}^{3}\right)$. "P<0.05, Wilcoxon test. PLD, PEGylated liposomal doxorubicin; $\mathrm{BEV}$, bevacizumab; PTX, paclitaxel; 2L, second line.

maintenance treatment in the RMG-I xenograft model. Although the tumors regressed during the 4 weeks of bevacizumab maintenance treatment following paclitaxel plus bevacizumab induction treatment, when the maintenance treatment was continued further, tumor regrowth was observed in $61 / 110$ individuals at week 12 (data not shown). To investigate the efficacy of second-line treatment and the usefulness of continuous bevacizumab treatment following progression, at week 12 , mice whose tumors had regrown were selected and re-randomized into four groups for the second-line treatments. In the preliminary experiments, the sensitivity of treatmentnaive RMG-I tumors to PEGylated liposomal doxorubicin and gemcitabine was tested among the second-line chemotherapeutics used in the treatment of patients with ovarian cancer, and it 
A

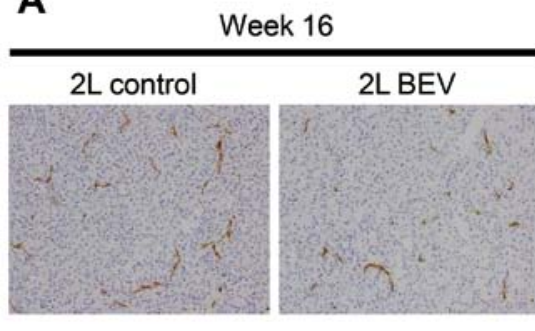

B

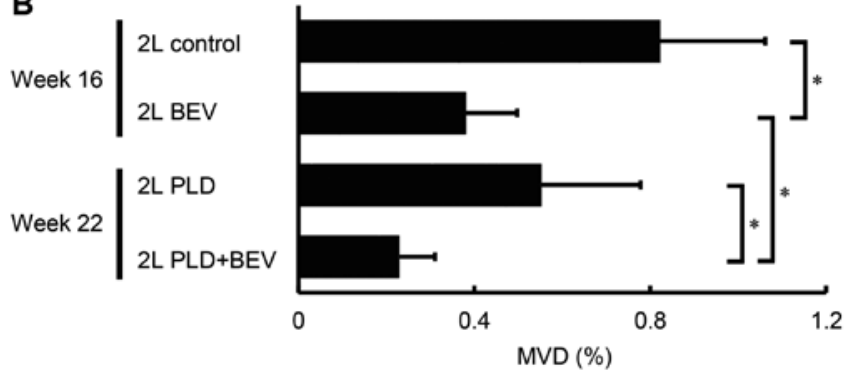

was determined that RMG-I was sensitive only to PEGylated liposomal doxorubicin (data not shown); therefore, this drug was selected for further experiments. PEGylated liposomal doxorubicin monotherapy was also efficacious in tumors that regrew during bevacizumab maintenance, and no significant tumor growth was observed from the initiation of treatment (weeks 22 vs. 13 in the second-line PEGylated liposomal doxorubicin treatment group; Fig. 4). Notably, the tumors in the second-line PEGylated liposomal doxorubicin plus bevacizumab treatment group regressed significantly from their volume at the initiation of this treatment (week 22 vs. 13). The tumor volume in the second-line PEGylated liposomal doxorubicin plus bevacizumab treatment group at week 22 was significantly reduced, compared with the tumor volume in the second-line PEGylated liposomal doxorubicin treatment group.

Anti-angiogenic activity of second-line PEGylated liposomal doxorubicin plus bevacizumab treatment following paclitaxel plus bevacizumab induction treatment and bevacizumab maintenance treatment in the RMG-I xenograft model. The investigation of MVD comparing second-line control treatment and second-line bevacizumab treatment was terminated at week 16 due to the tumor blister breaking in $3 / 7$ mice in the control group (the number pre-specified in the study protocol). MVD in the second-line bevacizumab treatment group was significantly lower, compared with the second-line control treatment group, on week 16, 4 days after the tumor blister breakage was observed (Fig. 5). Furthermore, MVD in the second-line PEGylated liposomal doxorubicin plus bevacizumab treatment group on week 22 was significantly lower, compared with in the second-line bevacizumab treatment group on week 16 and in the second-line PEGylated liposomal doxorubicin treatment group on week 22, indicating that the MVD suppression effect of the second-line bevacizumab was enhanced by the combination with the second-line PEGylated liposomal doxorubicin.

IGFBP-3 expression level in the RMG-I xenograft model. To investigate the mechanism of the further MVD reduction observed in the combination treatment, expression levels of
Week 22

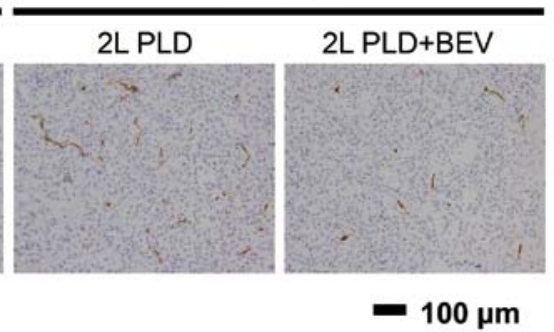

Figure 5. Anti-angiogenic activity of 2L PLD plus BEV treatment following PTX plus BEV induction treatment and BEV maintenance treatment in the RMG-I xenograft model. (A) Tumor microvessels stained immunohistochemically with antibody against CD31 on weeks 16 and 22. (B) The MVD in tumor tissue was determined by calculating the ratio of CD31-positive area to the total observed area. The dosage of PLD, BEV and PTX was 20,5 and $80 \mathrm{mg} / \mathrm{kg}$, respectively. Data represent the mean + standard deviation $(n=5-7) .{ }^{*} \mathrm{P}<0.05$, Wilcoxon test with Holm-Bonferroni correction. PLD, PEGylated liposomal doxorubicin; BEV, bevacizumab; PTX, paclitaxel; 2L, second line; MVD, microvessel density.

human angiogenesis factors in RMG-I tumor tissue were evaluated by a human angiogenesis array on samples obtained on weeks 16 and 22. Among the 55 angiogenesis-associated proteins on the array, only IGFBP-3 expression was affected, which was lower in the second-line PEGylated liposomal doxorubicin plus bevacizumab treatment group, compared with the second-line control, bevacizumab or PEGylated liposomal doxorubicin treatment groups (Fig. 6A). This tendency was confirmed with a quantitative ELISA assay of IGFBP-3 to be statistically significant (Fig. 6B). No reduced level in IGFBP-3 expression was observed in the second-line bevacizumab treatment or the second-line PEGylated liposomal doxorubicin treatment groups, compared with the IGFBP-3 expression in the second-line control group.

\section{Discussion}

The present study was conducted to address the following two clinical questions regarding continuous treatment with bevacizumab in ovarian cancer. The first question arises from the study reported by Burger et al (GOG-0218) (9) concerning the efficacy of bevacizumab maintenance following induction therapy with carboplatin and paclitaxel plus bevacizumab in ovarian clear cell carcinoma. The second question concerns the efficacy and mechanisms of bevacizumab beyond progression, which is continuous treatment with bevacizumab in combination with a second-line chemotherapeutic agent for patients who have disease progression after first-line chemotherapy plus bevacizumab combination therapy followed by maintenance with bevacizumab (11). Therefore, experiments were conducted using mouse xenograft models inoculated with the human ovarian clear cell carcinoma cell line RMG-I and the ovarian serous adenocarcinoma cell line SK-OV-3.

With respect to the first question, it was demonstrated that the regimens with bevacizumab maintenance treatment following induction treatments, including bevacizumab, exhibited an increased antitumor effect, compared with the regimens without bevacizumab, during the induction and maintenance phases in the SK-OV-3 and RMG-I xenograft models (Fig. 1). These results demonstrated that the regimens with bevacizumab 
A

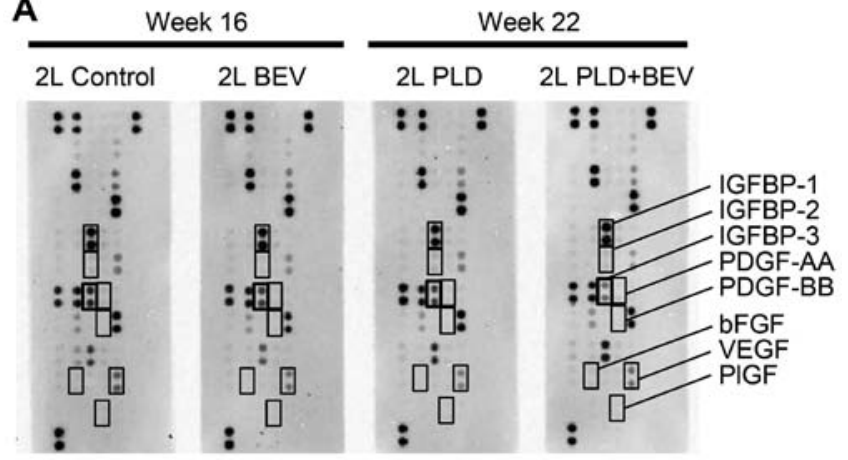

B

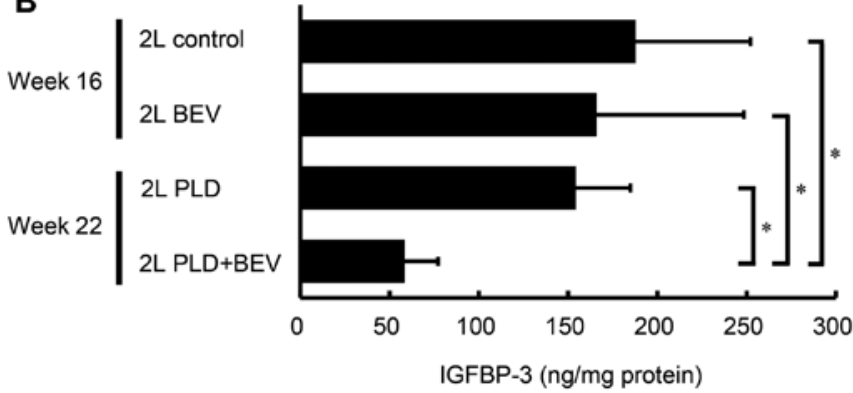

Figure 6. IGFBP-3 expression level in RMG-I xenografts treated with 2L PLD plus BEV following PTX plus BEV induction treatment and BEV maintenance treatment. (A) Expression levels of human angiogenesis factors in RMG-I tumor tissues were evaluated by human angiogenesis array on weeks 16 and 22. (B) IGFBP-3 expression level was determined by human ELISA assay on weeks 16 and 22. The dosage of PLD, BEV and PTX was 20,5 and $80 \mathrm{mg} / \mathrm{kg}$, respectively. Data represent the mean + standard deviation $(n=5-7)$. ${ }^{*} \mathrm{P}<0.05$, Wilcoxon test with Holm-Bonferroni correction. 2L, second line; PLD, PEGylated liposomal doxorubicin; BEV, bevacizumab; PTX, paclitaxel. IGFBP-3, insulin like growth factor binding protein-3; PDGF, platelet derived growth factor; bFGF, basic fibroblast growth factor; VEGF, vascular endothelial cell growth factor; PIGF, placental growth factor.

treatment throughout the induction and maintenance phases result in an increased antitumor effect, compared with regimens without bevacizumab, not only in ovarian serous carcinoma model, but also in the ovarian clear cell carcinoma model.

Notably, compared with the control maintenance treatment following control induction treatment group on week 6, the MVD was significantly lower, not only with the bevacizumab maintenance treatment following bevacizumab induction treatment group, but also with the control maintenance treatment following paclitaxel induction treatment group (Fig. 2). Additionally, the MVD with bevacizumab maintenance treatment following paclitaxel plus bevacizumab induction treatment group was even more significantly lower. Paclitaxel has been reported to exhibit anti-angiogenic activity through inhibition of proliferation, chemotaxis, chemo-invasion, alignment and cord formation of vascular endothelial cells $(20,21)$. Although the precise mechanisms of paclitaxel-induced anti-angiogenic activity have not yet been elucidated, the combination effect of paclitaxel and bevacizumab demonstrated in the present study indicates that these two agents act to reduce MVD via different underlying mechanisms, and this stronger MVD reduction is considered to contribute, at least in part, to superior antitumor activity of this treatment regimen.

Subsequently, the efficacy of continuous treatment with bevacizumab in the induction and maintenance phases in the clear cell carcinoma model was investigated. As expected, an increased antitumor activity was observed in the bevacizumab maintenance following paclitaxel plus bevacizumab treatment group, compared with the groups without bevacizumab maintenance (Fig. 3A). In additional experiments, increased antitumor activity was observed with bevacizumab maintenance treatment following paclitaxel plus bevacizumab induction treatment, compared with bevacizumab maintenance treatment following paclitaxel induction treatment (Fig. 3B). These data indicated that bevacizumab treatment was notable not only during the induction phase in combination with paclitaxel, but also during the maintenance phase in the ovarian clear cell carcinoma model. The MVD following bevacizumab maintenance treatment following paclitaxel plus bevacizumab induction treatment was significantly lower at week 9, compared with the MVD following paclitaxel plus bevacizumab induction treatment without maintenance treatment (Fig. 3C and D). Collectively, this data indicated that continuous presence of bevacizumab is required to maintain the tumor microvessel density at a low level, and the addition of paclitaxel during induction further augments the reduction of these vessels.

With respect to the second question, since the efficacy and mechanism of bevacizumab beyond progression has not been demonstrated in ovarian cancer, to investigate its potency in a non-clinical model, mice whose tumors exhibited regrowth during bevacizumab maintenance were selected and PEGylated liposomal doxorubicin was used as a second-line partner of bevacizumab in the RMG-I xenograft model. Second-line PEGylated liposomal doxorubicin plus bevacizumab exhibited a significantly increased antitumor activity, compared with second-line PEGylated liposomal doxorubicin alone, even when bevacizumab monotherapy itself had no apparent effect on tumor growth (Fig. 4).

This result raises two possibilities. Firstly, continuous inhibition of VEGF-A by bevacizumab was still required to reduce angiogenesis, but reduction of angiogenesis alone was insufficient to control tumor growth at this stage, unlike in the treatment naive stage in this model, but it was efficacious when combined with PEGylated liposomal doxorubicin. The other possibility is that RMG-I had acquired resistance to bevacizumab by VEGF-Aindependent angiogenesis, which was blocked by PEGylated liposomal doxorubicin to produce the combination effect. We previously reported that the latter mechanism was observed with capecitabine in a human colon cancer cell xenograft model after acquired resistance to bevacizumab (22).

To investigate the mechanism of action of second-line bevacizumab with PEGylated liposomal doxorubicin, MVD in RMG-I tumors was analyzed. Compared with control in the second-line treatment, bevacizumab kept MVD significantly lower, which indicated that tumor angiogenesis remained dependent on VEGF-A and that resistance to bevacizumab had not been acquired, at least in terms of angiogenesis in this model. Notably, the bevacizumab-induced decrease in MVD was significantly enhanced by PEGylated liposomal doxorubicin (Fig. 5), indicating that this drug may also have additional VEGF-A-independent suppressive effects on angiogenesis.

Among the angiogenesis-associated proteins that were tested, IGFBP-3 was the only one where the expression was determined to be lower when bevacizumab was combined with PEGylated liposomal doxorubicin. Additionally, no changes in 
the expression levels of IGFBP-1 or IGFBP-2, which belong to the same protein family as IGFBP-3, or other angiogenic factors, including basic fibroblast growth factor, placental growth factor, platelet derived growth factor (PDGF)-AA and PDGF-BB, were observed (Fig. 6A). Granata et al (23) demonstrated that IGFBP-3 induces angiogenesis in an in vitro assay of capillarylike structure formation and in a quantitative in vivo analysis of neovascularization in Matrigel plugs. Although PEGylated liposomal doxorubicin alone and bevacizumab alone did not affect human IGFBP-3 expression, PEGylated liposomal doxorubicin plus bevacizumab treatment resulted in levels of human IGFBP-3 being significantly lower, compared with levels following PEGylated liposomal doxorubicin alone or bevacizumab alone treatment (Fig. 6B).

It was reported that IGFBP-3 secretion is stimulated by VEGF, and IGFBP-3 and VEGF secretions are induced by hypoxia inducible factor (HIF)-1 $\alpha(24,25)$. Accordingly, in the present model, although IGFBP-3 production by VEGF is considered to be inhibited by treatment with bevacizumab alone, IGFBP-3 production via HIF-1 $\alpha$ derived from hypoxia may be enhanced and act to cancel out any change of IGFPB-3. However, doxorubicin is reported to inhibit the activation of HIF-1 $\alpha$ (26); therefore, when PEGylated liposomal doxorubicin and bevacizumab are combined, simultaneous inhibition of VEGF and HIF-1 $\alpha$ may result in a decrease in the production of IGFBP-3. In addition to the blockade of VEGF by bevacizumab, the decrease in IGFBP-3 by PEGylated liposomal doxorubicin plus bevacizumab may contribute to the continuous and enhanced suppression of MVD and may be the mechanism through which bevacizumab beyond progression acts in the present model.

It is not probable that the disease progression observed in the present study is resistance to bevacizumab due to increased IGFBP-3 expression, since there was no difference in level of IGFBP-3 expression between the second-line control group and the second-line bevacizumab group. The precise mechanism by which the PEGylated liposomal doxorubicin plus bevacizumab combination reduces the IGFBP-3 level and its general contribution to tumor angiogenesis remains to be elucidated. Although one of the proposed modes of action of bevacizumab is to increase the intra-tumor concentration of co-administered chemotherapeutics (27), the present study confirmed that this was not the case since there was no difference in apoptosis, number of cells in the $\mathrm{M}$ phase or Ki-67 levels, due to bevacizumab induction, maintenance or second-line treatment (data not shown).

In conclusion, the present study demonstrated that bevacizumab maintenance treatment following paclitaxel plus bevacizumab induction treatment produced antitumor activity superior to that with no bevacizumab treatment as induction or maintenance in ovarian clear cell carcinoma. Additionally, it indicated that second-line PEGylated liposomal doxorubicin plus bevacizumab treatment following paclitaxel plus bevacizumab induction and bevacizumab maintenance treatment had antitumor activity superior to that with second-line PEGylated liposomal doxorubicin alone following the same induction and maintenance treatments. Bevacizumab treatment throughout induction, maintenance and the second-line combination phase resulted in a continuous reduction of MVD, which indicates that it is the primary mechanism underlying the superior efficacy in the first-line induction, maintenance and second-line treatments that include bevacizumab. Further studies are required to elucidate the efficacy and the mechanism of action of the continuation of bevacizumab as a maintenance therapy following induction treatment including bevacizumab, and as a bevacizumab beyond progression, in ovarian cancer.

\section{Acknowledgements}

The authors would like to thank Dr Kaori Fujimoto-Ouchi for her advice on experimental design, and Ms. Masako Miyazaki and Ms. Hiromi Sawamura for their excellent technical assistance (Product Research Department, Chugai Pharmaceutical Co., Ltd. Kamakura, Japan).

\section{Funding}

No funding was received.

\section{Availability of data and materials}

The datasets analyzed during the study are available from the corresponding author on reasonable request.

\section{Authors' contributions}

NI designed and performed the experiments, analyzed the data, and drafted the manuscript. KYo and MK produced the slides, performed the pathological diagnosis, and analyzed the data. MY collected the data. MS performed data interpretation and revised the manuscript. KYa provided the study concept and design. All authors have read and approved the final manuscript.

\section{Ethics approval and consent to participate}

All animal experiments were reviewed and approved by the Institutional Animal Care and Use Committee at Chugai Pharmaceutical Co., Ltd. (approval no. 13-225, 15-201).

\section{Patient consent for publication}

Not applicable.

\section{Competing interests}

The authors declare that they have no competing interests.

\section{References}

1. Ferlay J, Soerjomataram I, Dikshit R, Eser S, Mathers C, Rebelo M, Parkin DM, Forman D and Bray F: Cancer incidence and mortality worldwide: Sources, methods and major patterns in GLOBOCAN 2012. Int J Cancer 136: E359-E386, 2015.

2. Chan JK, Teoh D, Hu JM, Shin JY, Osann K and Kapp DS: Do clear cell ovarian carcinomas have poorer prognosis compared to other epithelial cell types? A study of 1411 clear cell ovarian cancers. Gynecol Oncol 109: 370-376, 2008.

3. Sugiyama T, Kamura T, Kigawa J, Terakawa N, Kikuchi Y, Kita T, Suzuki M, Sato I and Taguchi K: Clinical characteristics of clear cell carcinoma of the ovary: A distinct histologic type with poor prognosis and resistance to platinum-based chemotherapy. Cancer 88: 2584-2589, 2000.

4. Itamochi H, Kigawa J and Terakawa N: Mechanisms of chemoresistance and poor prognosis in ovarian clear cell carcinoma. Cancer Sci 99: 653-658, 2008. 
5. Mackay HJ, Brady MF, Oza AM, Reuss A, Pujade-Lauraine E, Swart AM, Siddiqui N, Colombo N, Bookman MA, Pfisterer J, Du Bois A; Gynecologic Cancer InterGroup: Prognostic relevance of uncommon ovarian histology in women with stage III/IV epithelial ovarian cancer. Int J Gynecol Cancer 20: 945-952, 2010.

6. Piccart MJ, Bertelsen K, James K, Cassidy J, Mangioni C, Simonsen E, Stuart G, Kaye S, Vergote I, Blom R, et al: Randomized intergroup trial of cisplatin-paclitaxel versus cisplatin-cyclophosphamide in women with advanced epithelial ovarian cancer: Threeyear results. J Natl Cancer Inst 92: 699-708, 2000.

7. Vasey PA, Jayson GC, Gordon A, Gabra H, Coleman R, Atkinson R, Parkin D, Paul J, Hay A and Kaye SB; Scottish Gynaecological Cancer Trials Group: Phase III randomized trial of docetaxelcarboplatin versus paclitaxel-carboplatin as first-line chemotherapy for ovarian carcinoma. J Natl Cancer Inst 96: 1682-1691, 2004.

8. Köbel M, Kalloger SE, Huntsman DG, Santos JL, Swenerton KD, Seidman JD, Gilks CB; Cheryl Brown Ovarian Cancer Outcomes Unit of the British Columbia Cancer Agency, Vancouver BC: Differences in tumor type in low-stage versus high-stage ovarian carcinomas. Int J Gynecol Pathol 29: 203-211, 2010.

9. Burger RA, Brady MF, Bookman MA, Fleming GF, Monk BJ, Huang H, Mannel RS, Homesley HD, Fowler J, Greer BE, et al Gynecologic Oncology Group: Incorporation of bevacizumab in the primary treatment of ovarian cancer. N Engl J Med 365 : 2473-2483, 2011.

10. Morgan RJ Jr, Armstrong DK, Alvarez RD, Bakkum-Gamez JN, Behbakht K, Chen LM, Copeland L, Crispens MA, De Rosa M Dorigo O, et al: Ovarian Cancer, Version 1.2016, NCCN Clinical Practice Guidelines in Oncology. J Natl Compr Canc Netw 14: 1134-1163, 2016.

11. Masi G, Salvatore L, Boni L, Loupakis F, Cremolini C, Fornaro L, Schirripa M, Cupini S, Barbara C, Safina V, et al; BEBYP Study Investigators: Continuation or reintroduction of bevacizumab beyond progression to first-line therapy in metastatic colorectal cancer: Final results of the randomized BEBYP trial. Ann Oncol 26: 724-730, 2015

12. Presta LG, Chen H, O'Connor SJ, Chisholm V, Meng YG, Krummen L, Winkler M and Ferrara N: Humanization of an anti-vascular endothelial growth factor monoclonal antibody for the therapy of solid tumors and other disorders. Cancer Res 57 4593-4599, 1997.

13. Braghiroli MI, Sabbaga J and Hoff PM: Bevacizumab: Overview of the literature. Expert Rev Anticancer Ther 12: 567-580, 2012.

14. Crossin KL and Carney DH: Evidence that microtubule depolymerization early in the cell cycle is sufficient to initiate DNA synthesis. Cell 23: 61-71, 1981.

15. Gabizon AA: Pegylated liposomal doxorubicin: Metamorphosis of an old drug into a new form of chemotherapy. Cancer Invest 19 424-436, 2001
16. Pérez-López ME, Curiel T, Gómez JG and Jorge M: Role of pegylated liposomal doxorubicin (Caelyx) in the treatment of relapsing ovarian cancer. Anticancer Drugs 18: 611-617, 2007.

17. Fogh J, Wright WC and Loveless JD: Absence of HeLa cell contamination in 169 cell lines derived from human tumors. J Natl Cancer Inst 58: 209-214, 1977.

18. Nozawa S, Tsukazaki K, Sakayori M, Jeng $\mathrm{CH}$ and Iizuka R: Establishment of a human ovarian clear cell carcinoma cell line (RMG-I) and its single cell cloning - with special reference to the stem cell of the tumor. Hum Cell 1: 426-435, 1988.

19. Holm S: A simple sequentially rejective multiple test procedure. Scand J Stat 6: 65-70, 1979

20. Belotti D, Vergani V, Drudis T, Borsotti P, Pitelli MR, Viale G, Giavazzi $\mathrm{R}$ and Taraboletti G: The microtubule-affecting drug paclitaxel has antiangiogenic activity. Clin Cancer Res 2: 1843-1849, 1996.

21. Naumova E, Ubezio P, Garofalo A, Borsotti P, Cassis L, Riccardi E, Scanziani E, Eccles SA, Bani MR and Giavazzi R: The vascular targeting property of paclitaxel is enhanced by SU6668, a receptor tyrosine kinase inhibitor, causing apoptosis of endothelial cells and inhibition of angiogenesis. Clin Cancer Res 12: 1839-1849, 2006.

22. Iwai T, Sugimoto M, Harada S, Yorozu K, Kurasawa $M$ and Yamamoto K: Continuous administration of bevacizumab plus capecitabine, even after acquired resistance to bevacizumab, restored anti-angiogenic and antitumor effect in a human colorectal cancer xenograft model. Oncol Rep 36: 626-632, 2016.

23. Granata R, Trovato L, Lupia E, Sala G, Settanni F, Camussi G, Ghidoni R and Ghigo E: Insulin-like growth factor binding protein-3 induces angiogenesis through IGF-I- and SphK1-dependent mechanisms. J Thromb Haemost 5: 835-845, 2007.

24. Slomiany MG and Rosenzweig SA: Autocrine effects of IGF-Iinduced VEGF and IGFBP-3 secretion in retinal pigment epithelial cell line ARPE-19. Am J Physiol Cell Physiol 287: C746-C753, 2004.

25. Slomiany MG and Rosenzweig SA: IGF-1-induced VEGF and IGFBP-3 secretion correlates with increased HIF-1 alpha expression and activity in retinal pigment epithelial cell line D407. Invest Ophthalmol Vis Sci 45: 2838-2847, 2004.

26. Duyndam MC, van Berkel MP,Dorsman JC, Rockx DA, Pinedo HM and Boven E: Cisplatin and doxorubicin repress vascular endothelial growth factor expression and differentially down-regulate hypoxia-inducible factor I activity in human ovarian cancer cells. Biochem Pharmacol 74: 191-201, 2007.

27. Yanagisawa M, Yorozu K, Kurasawa M, Nakano K, Furugaki K, Yamashita Y, Mori K and Fujimoto-Ouchi K: Bevacizumab improves the delivery and efficacy of paclitaxel. Anticancer Drugs 21: 687-694, 2010.

This work is licensed under a Creative Commons Attribution-NonCommercial-NoDerivatives 4.0 International (CC BY-NC-ND 4.0) License. 